ANNALES

POLONICI MATHEMATICI

$55(1991)$

\title{
Supercomplex structures, surface soliton equations, and quasiconformal mappings
}

\author{
by JULian ŁAWRYNOWiCz* (Łódź), KATARZYNA KJDZiA* (Łódź) \\ and Osamu Suzuki** (Tokyo) \\ Dedicated to Prof. K. Oikawa on his 65th birthday
}

\begin{abstract}
Hurwitz pairs and triples are discussed in connection with algebra, complex analysis, and field theory. The following results are obtained: (i) A field operator of Dirac type, which is called a Hurwitz operator, is introduced by use of a Hurwitz pair and its characterization is given (Theorem 1). (ii) A field equation of the elliptic Neveu-Schwarz model of superstring theory is obtained from the Hurwitz pair $\left(\mathbb{E}^{4}, \mathbb{E}^{3}\right)$ (Theorem 2), and its counterpart connected with the Hurwitz triple $\left(\mathbb{E}^{11}, \mathbb{E}^{11}, \mathbb{E}^{26}\right)$ is mentioned. (iii) Isospectral deformations of the Hurwitz operator of the Hurwitz pair $\left(\mathbb{E}^{2}, \mathbb{E}^{2}\right)$ induce various soliton equations (Theorem 3). (iv) A special complex structure, which is called a supercomplex structure, is introduced on separable Hilbert spaces (Definition 10). A correspondence between such structures and reduction solutions of Sato's version of Kadomtsev-Petviashvili system is established (Theorem 4). (v) The general class of quasiconformal mappings in the plane is obtained from generalized Hurwitz pairs (Theorem 5). From these results we conclude that Hurwitz pairs and triples give rise to several interesting applications.
\end{abstract}

Introduction. This paper is the third part of our study of Hurwitz pairs. In the first part [11], we gave an outline of a field theory defined by Hurwitz pairs. We introduced a field equation of Dirac type and soliton equations by means of Hurwitz pairs. In the second paper [12] the concepts of a Hurwitz pair and the corresponding supercomplex structure were considered in a slightly more general setting of weighted Hurwitz pairs. Then, as applications, several results were obtained for field equations with mass and for Beltrami equations generating quasiconformal mappings in the plane

1991 Mathematics Subject Classification: 15A63, 35Q51, 30C60.

* Research supported by CPBP 01.08.

** The third-named author was supported by the Polish Academy of Sciences and the University of Łódź during the preparation of this work. 
with real-valued complex dilatation.

In this paper we give a systematic treatment of Hurwitz pairs in connection with algebra, complex analysis and field theory, indicating possible generalizations to the case of Hurwitz triples [3]. Our main concern is not to carry out a detailed study but only to suggest relationships between Hurwitz pairs or triples and other areas. Thus we shall restrict our considerations mainly to the simplest Hurwitz pair $\left(\mathbb{E}^{2}, \mathbb{E}^{2}\right), \mathbb{E}^{2}$ denoting the two-dimensional Euclidean space, and state our results in a simplified form. Our main results are Theorems $1-5$. The heart of this paper is Sections 5-8. The first three sections supply preliminaries.

The following ones are some recent works on Hurwitz pairs. The secondnamed author's study on supercomplex structures, joint with J. Rembieliński ([20], [21]), provides a quite detailed guide to Hurwitz pairs. For Hurwitz pairs of pseudo-euclidean spaces, we refer to [22] and [14]. In [14], the Minkowski space is discussed in connection with Hurwitz pairs. In [13], low dimensional Hurwitz pairs are discussed by use of the multiplication structure of octonions.

In 1898 and 1923, A. Hurwitz [9], [10] discovered very mysterious pairs $\left(\mathbb{E}^{n}, \mathbb{E}^{p}\right)$ of Euclidean spaces $\mathbb{E}^{n}$ and $\mathbb{E}^{p}$, which we call Hurwitz pairs. We understand that he introduced them in connection with the composition problem of quadratic forms in number theory. Fifty years later A. Andreotti took interest in Hurwitz pairs in connection with field theory because a special class of Clifford algebras is generated by Hurwitz pairs. He proposed to the first-named author the problem of characterizing Hurwitz pairs in terms of field theory. This gave a strong motivation for further research on Hurwitz pairs. In fact, the first-named author and J. Rembieliński discussed Hurwitz pairs within the modern framework of the theory of Clifford algebras and introduced the notion of supercomplex structure [20], [21].

In [11], we introduced a field operator of Dirac type by use of specific properties of the Clifford algebras which are defined by Hurwitz pairs. These operators were called Hurwitz operators and the corresponding equations Hurwitz equations. We gave a characterization of Hurwitz pairs in terms of Hurwitz operators. This may be regarded as an answer to the question posed by Andreotti.

In the present article only Euclidean spaces are considered. We take Hurwitz equations as a starting point for our research and wander in various directions (Section 4). To discuss complex analysis in terms of Hurwitz pairs, we regard our equations as defining equations of (generalized) holomorphic functions in the sense of Clifford analysis. Our equations are equivalent to the Cauchy-Riemann equations in the case of the Hurwitz pair $\left(\mathbb{E}^{2}, \mathbb{E}^{2}\right)$ and to the Fueter equation in the case of the Hurwitz pair $\left(\mathbb{E}^{4}, \mathbb{E}^{4}\right)$ (Proposition 7). In order to discuss Hurwitz pairs in connection with field 
theory, it suffices to consider the Hurwitz equations alone. In fact, in the case of $\left(\mathbb{E}^{4}, \mathbb{E}^{4}\right)$, the Hurwitz equation is nothing but the non-relativistic Dirac equation and in the case of $\left(\mathbb{E}^{4}, \mathbb{E}^{3}\right)$ it gives rise to the field equations which appear in the elliptic Neveu-Schwarz model of superstring theory (Theorem 1 in Section 4 and Theorem 2 in Section 5). Now, according to the modern setting [8], it is natural to introduce the Hurwitz triples [3] and extend the theory to the triple $\left(\mathbb{E}^{11}, \mathbb{E}^{11}, \mathbb{E}^{26}\right) ;$ cf. $[2]$.

To discuss the soliton equations in connection with Hurwitz pairs, we consider the isospectral deformations of Hurwitz operators (Section 6). The Hurwitz operator of the Hurwitz pair $\left(\mathbb{E}^{2}, \mathbb{E}^{2}\right)$ is equivalent to that of Ablowitz et al. [1]. Thus we can easily see that various soliton equations are obtained by use of our isospectral deformations (Theorem 3 in Section 6). We discuss the Kadomtsev-Petviashvili system for the Hurwitz operator and obtain its linearization equation (Theorem 3). Next we arrive at a result which yields a relationship between complex analysis and soliton equations. We generalize the concept of a pre-Hurwitz pair to a pair $\left(\mathbb{H}, \mathbb{E}^{p}\right)$ of a separable Hilbert space $\mathbb{H}$ and the Euclidean space $\mathbb{E}^{p}$, and define supercomplex structures on $\mathbb{H}$. Then we prove that there exists a correspondence between the supercomplex structures of a separable Hilbert space and the reduction solutions of the original Kadomtsev-Petviashvili system (Theorem 4 in Section 7). Section 8 gives a new description of the full class of quasiconformal mappings in an arbitrary domain in the complex plane in terms of distributional solutions of certain weighted Fueter equations, where an important role is played by the method of isospectral deformations [30] which may be considered a generalization of the parametrical method for quasiconformal mappings in the plane [19].

We may conclude that Hurwitz pairs and triples have many connections with various fields of mathematics and physics, and one may expect further development of the theory.

The third-named author would like to express his hearty thanks to Profs. S. Sakai, J. Yamashita and T. Mukasa, and to Drs. M. Jimbo and S. Kanemaki for numerous discussions and suggestions.

1. Hurwitz pairs. In this section we recall some basic facts on Hurwitz pairs and triples. A. Hurwitz considered the following problem: Find a pair $\left(\mathbb{E}^{n}, \mathbb{E}^{p}\right)$ of the $n$-dimensional and $p$-dimensional Euclidean spaces $\mathbb{E}^{n}$ and $\mathbb{E}^{p}$ which admits a bilinear mapping $f: \mathbb{E}^{n} \times \mathbb{E}^{p} \rightarrow \mathbb{E}^{n}$ with the following property:

$$
\|f(x, y)\|=\|x\|\|y\| \quad \text { for any } x \in \mathbb{E}^{n} \text { and } y \in \mathbb{E}^{p},
$$

where \|\| denotes the usual norms of the Euclidean spaces. This condition is called the Hurwitz condition and the mapping $f$ a Hurwitz mapping. Hurwitz 
discovered that such pairs are strongly restricted. We examine some simple cases. The pair $\left(\mathbb{E}^{1}, \mathbb{E}^{1}\right)$ satisfies $(1)$ for $f(x, y)=x y$. For any pair of complex numbers $z, w \in \mathbb{C}$,

$$
|z w|=|z||w| \text {. }
$$

Hence $\left(\mathbb{E}^{2}, \mathbb{E}^{2}\right)$ satisfies (1). In a similar manner, we see that (2) holds for any pair of quaternions or octonions. Hence $\left(\mathbb{E}^{4}, \mathbb{E}^{4}\right)$ and $\left(\mathbb{E}^{8}, \mathbb{E}^{8}\right)$ satisfy (1). If a pair $\left(\mathbb{E}^{n}, \mathbb{E}^{p}\right)$ satisfies $(1)$, then so does $\left(\mathbb{E}^{n} \oplus \mathbb{E}^{n}, \mathbb{E}^{p}\right)$. Thus we have to introduce the concept of irreducibility: $f$ is called irreducible if $f$ does not preserve any non-trivial proper subspace $V$ of $\mathbb{E}^{n}$, i.e., there exists no non-trivial subspace $V$ of $\mathbb{E}^{n}$ which satisfies

$$
\left.f\right|_{V \times \mathbb{E}^{p}}: V \times \mathbb{E}^{p} \rightarrow V .
$$

Then we introduce the following definition:

Definition 1. A pair of Euclidean spaces $\mathbb{E}^{n}$ and $\mathbb{E}^{p}$ is called a preHurwitz pair if there exists a bilinear mapping $f: \mathbb{E}^{n} \times \mathbb{E}^{p} \rightarrow \mathbb{E}^{n}$ which satisfies (1). Moreover, if $f$ is irreducible, the pair is called a Hurwitz pair.

Hurwitz [9] proved the following

Proposition 1. $\left(\mathbb{E}^{n}, \mathbb{E}^{n}\right)$ is a Hurwitz pair if and only if $n=1,2,4$, or 8 .

More generally, he arrived at [10]

Proposition 2. If $\left(\mathbb{E}^{n}, \mathbb{E}^{p}\right)$ is a Hurwitz pair $(n \geq p)$, then only the following $(n, p)$ are possible: $(n, p)=(1,1)$ and for $s=0,1,2, \ldots$,
(i) $n=2^{r}$
and $p=2 r$, $r=4 s+1$ or $4 s+3$,
(ii) $n=2^{r}$
and $p=2 r$,
$r=4 s+2$,
(iii) $n=2^{r-1}$
and $p=2 r$,
$r=4 s+4$,
(iv) $n=2^{r+1}$
and $p=2 r+1$
$r=4 s+1$
or $4 s+2$,
(v) $n=2^{r}$
and $p=2 r$,
$r=4 s+3$ or $4 s+4$.

The first possible pairs $(n, p)$ are listed as follows:
$(1,1) \quad(8,5) \quad(16,9) \quad(128,13)$
$(2,2) \quad(8,6) \quad(32,10) \quad(128,14)$
$(4,3) \quad(8,7) \quad(64,11) \quad(128,15)$
$(4,4) \quad(8,8) \quad(64,12) \quad(128,16)$

The table (i)-(v) in Proposition 2 suggests that $\mathbb{E}^{n}$ is the representation space of the Clifford algebra defined by $\mathbb{E}^{p}$, which was discovered by Hurwitz [10]. In order to get the relationship with the Clifford algebra, we write down the condition (1) using orthonormal bases $e_{1}, \ldots, e_{n}$ of $\mathbb{E}^{n}$ and 
$\varepsilon_{1}, \ldots, \varepsilon_{p}$ of $\mathbb{E}^{p}$. We define $n \times n$-matrices

$$
C_{\alpha}=\left(c_{j \alpha}^{k}\right), \quad \alpha=1, \ldots, p, \quad \text { by } \quad f\left(e_{j}, \varepsilon_{\alpha}\right)=\sum_{k=1}^{n} c_{j \alpha}^{k} e_{k} .
$$

Then the Hurwitz mapping can be written in the form

$$
f(x, y)=f(y) x, \quad f(y)=y_{1} C_{1}+\ldots+y_{p} C_{p} .
$$

We can prove (cf. [20]) the following

Proposition 3. The mapping $f$ in (4) satisfies the Hurwitz condition (1) if and only if

$$
C_{\alpha}^{T} C_{\beta}+C_{\beta}^{T} C_{\alpha}=2 \delta_{\alpha \beta} I_{n}, \quad \alpha, \beta=1, \ldots, p,
$$

where $I_{n}$ denotes the identity matrix and $\delta_{\alpha \beta}$ is the Kronecker symbol.

Corollary 1. $C_{\alpha}, \alpha=1, \ldots, p$, are orthogonal matrices.

Using Proposition 3 we can prove

Lemma 1. Let $\gamma_{\alpha}, \alpha=1, \ldots, p-1$, be complex matrices defined by $C_{\alpha}=i C_{p} \gamma_{\alpha}$. Then

$$
\begin{gathered}
\gamma_{\alpha} \gamma_{\beta}+\gamma_{\beta} \gamma_{\alpha}=2 \delta_{\alpha \beta} I_{n}, \quad \alpha, \beta=1, \ldots, p-1, \\
\gamma_{\alpha}^{T}=-\gamma_{\alpha} \quad \text { and } \quad \operatorname{re} \gamma_{\alpha}=0 .
\end{gathered}
$$

Hence we see that a pre-Hurwitz pair determines a Clifford algebra with the additional condition (7). Conversely, given a Clifford algebra generated by matrices $\gamma_{\alpha}$ satisfaying (7), we can obtain a pre-Hurwitz pair in the following manner: Choosing an arbitrary orthogonal matrix $C_{p}$, we define $C_{\alpha}, \alpha=1, \ldots, p-1$, by $C_{\alpha}=i C_{p} \gamma_{\alpha}$. Then the $C_{\alpha}$ satisfy (5). Using these matrices we define $f$ by (4). Then we obtain the desired Hurwitz mapping. Moreover, if we choose an irreducible Clifford algebra with (7), we obtain a Hurwitz pair. Therefore (cf. [20], [21]) we arrive at

Proposition 4. The determination of a Hurwitz pair is equivalent to that of an irreducible Clifford algebra which admits a representation satisfying (7). Hence $(n, p)$ can be characterized by

$$
n= \begin{cases}2^{[p / 2-1 / 2]} & \text { for } p \equiv 0,1,7(\bmod 8), \\ 2^{[p / 2+1 / 2]} & \text { for } p \equiv 2,3,4,5,6(\bmod 8) .\end{cases}
$$

Finally, we make a remark on Hurwitz mappings. If $f$ is a Hurwitz mapping, then so is $C f$, where $C$ is an orthogonal matrix. Hence a Hurwitz mapping can be written in terms of the $\gamma_{\alpha}$ :

$$
f=-i y_{1} \gamma_{1}-i y_{2} \gamma_{2}-\ldots-i y_{p-1} \gamma_{p-1}+y_{p} I_{n} .
$$

2. Pseudo-euclidean Hurwitz triples (cf. [3]). Consider three finitedimensional real vector spaces $S, V$ and $W$, equipped with non-degenerate 
pseudo-euclidean or symplectic real scalar products $(,)_{S},(,)_{V}$ and $(,)_{W}$. More precisely,

$$
\begin{aligned}
& (a, b)_{S} \in \mathbb{R}, \quad(b, a)_{S}=(a, b)_{S}, \quad(\gamma a, b)_{S}=\gamma(a, b)_{S}, \\
& (a, b+c)_{S}=(a, b)_{S}+(a, c)_{S}
\end{aligned}
$$

for $a, b, c, \in S, \gamma \in \mathbb{R}$;

$$
\begin{aligned}
& (f, g)_{V} \in \mathbb{R}, \quad(g, f)_{V}=\delta(f, g)_{V} \quad \text { with } \delta=1 \text { or }-1, \\
& (\gamma f, g)_{V}=\gamma(f, g)_{V}, \quad(f, g+h)_{V}=(f, g)_{V}+(f, h)_{V}
\end{aligned}
$$

for $f, g, h \in V, \gamma \in \mathbb{R}$;

$$
\begin{aligned}
& (F, G)_{W} \in \mathbb{R}, \quad(G, F)_{W}=\omega(F, G)_{W} \quad \text { with } \omega=1 \text { or }-1, \\
& (\gamma F, G)_{W}=\gamma(F, G)_{W}, \quad(F, G+H)_{W}=(F, G)_{W}+(F, H)_{W}
\end{aligned}
$$

for $F, G, H \in W, \gamma \in \mathbb{R}$.

In $S, V$ and $W$ we choose bases $\left(\varepsilon_{\alpha}\right),\left(e_{j}\right)$ and $\left(E_{A}\right)$, respectively, with

$$
\begin{aligned}
\alpha=1, \ldots, p & =\operatorname{dim} S, \quad j=1, \ldots, n=\operatorname{dim} V, \\
A & =1, \ldots, N=\operatorname{dim} W .
\end{aligned}
$$

Hence

$$
a=\sum_{\alpha=1}^{p} a^{\alpha} \varepsilon_{\alpha}, f=\sum_{j=1}^{n} f^{j} e_{j}, F=\sum_{A=1}^{N} F^{A} E_{A}, \quad \text { etc. }
$$

The metric tensors are

$$
\begin{gathered}
\eta \equiv\left[\eta_{\alpha \beta}\right]:=\left[\left(\varepsilon_{\alpha}, \varepsilon_{\beta}\right)_{S}\right], \quad \kappa \equiv\left[\kappa_{j k}\right]:=\left[\left(e_{j}, e_{k}\right)_{V}\right], \\
K \equiv\left[K_{A B}\right]:=\left[\left(E_{A}, E_{B}\right)_{W}\right],
\end{gathered}
$$

respectively. By (9)-(11) the tensors

$$
\eta^{-1} \equiv\left[\eta^{\alpha \beta}\right], \quad \kappa^{-1} \equiv\left[\kappa^{j k}\right], \quad K^{-1} \equiv\left[K^{A B}\right]
$$

exist and we have

$$
\eta^{T}=\eta, \quad \operatorname{det} \eta \neq 0, \quad \kappa^{T}=\delta \kappa, \quad \operatorname{det} \kappa \neq 0, \quad K^{T}=\omega K, \quad \operatorname{det} K \neq 0 .
$$

Without any loss of generality we can choose the basis $\left(\varepsilon_{\alpha}\right)$ so that

$$
\eta=\operatorname{diag}(1, \ldots, 1,-1, \ldots,-1), \quad \text { and hence } \eta^{-1}=\eta .
$$

In terms of metric tensors, the scalar products read:

$$
\begin{gathered}
(a, b)_{S}=\sum_{\alpha, \beta=1}^{p} \eta_{\alpha \beta} a^{\alpha} b^{\beta}, \quad(f, g)_{V}=\sum_{j, k=1}^{n} \kappa_{j k} f^{j} g^{k}, \\
(F, G)_{W}=\sum_{A, B=1}^{N} K_{A B} F^{A} G^{B} .
\end{gathered}
$$


By a Hurwitz mapping $\phi$ corresponding to $(W, V, S)$ we mean any mapping $S \times V \rightarrow W, \phi(a, f)=: a f$, with the properties:

$$
(a+b) f=a f+b f \quad \text { and } \quad a(f+g)=a f+a g,
$$

(13) $(a, a)_{S}(f, g)_{V}=(a f, a g)_{W} \quad$ (the generalized Hurwitz condition)

for $f, g \in V$ and $a, b \in S$. A straightforward verification leads to

LemMA 2. The generalized Hurwitz condition (13) implies $\delta=\omega . A$ Hurwitz mapping is uniquely determined by the "multiplication" scheme for base vectors:

$$
\varepsilon_{\alpha} e_{j}=\sum_{A=1}^{N} c_{j \alpha}^{A} E_{A} \quad \text { with } \alpha, j, A \text { as in }(12) .
$$

Corollary 2. The scheme (14), together with the postulates (9)-(11), yields, in particular, the following formulae for the real structure constants $c_{j \alpha}^{A}$ :

$$
c_{j \alpha}^{A}=\left(E^{A}, \varepsilon_{\alpha} e_{j}\right)_{W} \quad \text { with } \quad E^{A}:=\sum_{B=1}^{N} K^{A B} E_{B} .
$$

LEMMA 3. The generalized Hurwitz condition (14) is equivalent to each of the following conditions:

$$
\begin{gathered}
(a, b)_{S}(f, g)_{V}=\frac{1}{2}\left[(a f, b g)_{W}+(b f, a g)_{W}\right], \\
\left(a \eta a^{T}\right)(f \kappa g)^{T}=\gamma(a, f) K \gamma^{T}(a, g), \\
M(a) K M^{T}(a)=\eta\left(f \kappa g^{T}\right), \quad \text { where } f M(a)=\gamma(a, f), \\
N(f) K N^{T}(g)=\eta\left(f \kappa g^{T}\right), \quad \text { where } a N(f)=\gamma(a, f),
\end{gathered}
$$

for $f, g \in V$ and $a, b \in S$; here $\phi$ denotes the Hurwitz mapping $S \times V \rightarrow W$, and

$$
\begin{aligned}
& \phi^{T}=\left[\begin{array}{c}
\phi_{1} \\
\vdots \\
\phi_{N}
\end{array}\right], \quad g^{T}=\left[\begin{array}{c}
g_{1} \\
\vdots \\
g_{n}
\end{array}\right], \quad a^{T}=\left[\begin{array}{c}
a_{1} \\
\vdots \\
a_{p}
\end{array}\right], \\
& M(a)_{j}^{A}=\sum_{\alpha=1}^{n} c_{j \alpha}^{A} a^{\alpha}, \quad N(f)_{\alpha}^{A}=\sum_{j=1}^{n} c_{j \alpha}^{A} f^{j} .
\end{aligned}
$$

Lemmas 2 and 3 motivate the following

Definition 2. Any triple $(W, V, S)$ equipped with a Hurwitz mapping will be called a pseudo-euclidean Hurwitz triple.

A particularly important case appears when the Hurwitz triple $(W, V, S)$ is irreducible, i.e. when the Hurwitz mapping $\phi$ does not leave invariant 
proper subspaces of $(W, V)$ and their complements. More precisely, a Hurwitz triple $(W, V, S)$ is reducible whenever there are

(a) real vector subspaces $W_{1}$ and $W_{2}$ of $W, W_{1} \cap W_{2} \neq\{0\}$,

(b) real vector subspaces $V_{1}$ and $V_{2}$ of $V,\{0\} \neq V_{1} \neq V_{2}, V_{1} \oplus V_{2}=V$, such that $\phi\left[S \times V_{1}\right] \subset W_{1}, \phi\left[S \times V_{2}\right] \subset W_{2}$.

A Hurwitz triple $(W, V, S)$ is irreducible whenever it is not reducible.

If $W=V$ and there exists a unit element $\varepsilon_{0}$ in $S$ with respect to the Hurwitz mapping $S \times V \rightarrow V$, the Hurwitz triple reduces to a pseudoeuclidean pre-Hurwitz pair. If a Hurwitz triple $(W, V, S)$ is irreducible, $W=$ $V$, and there exists a unit element $\varepsilon_{0}$ as before, the Hurwitz triple reduces to a pseudo-euclidean Hurwitz pair.

For examples, we refer to [3].

3. Supercomplex structure. In this section we recall some basic facts on supercomplex structures. For details we refer to [20] and [21]. Let $\left(\mathbb{E}^{n}, \mathbb{E}^{p}\right)$ be a Hurwitz pair. Let $\gamma_{\alpha}, \alpha=1, \ldots, p-1$, be a system of matrices in Lemma 1 . We define $S_{\alpha}, \alpha=1, \ldots, p-1$, by

$$
S_{\alpha}=-i \gamma_{\alpha}, \quad \alpha=1, \ldots, p-1 .
$$

Then $S_{\alpha}^{2}=-I_{n}$. Hence $S_{\alpha}$ determines a complex structure in $\mathbb{E}^{n}$. Consider the $(p-2)$-dimensional sphere $S^{p-2}$. For $\widetilde{n} \in S^{p-2}, \widetilde{n}=\left(n_{1}, \ldots, n_{p-1}\right)$ with $n_{1}^{2}+\ldots+n_{p-1}^{2}=1$, we set

$$
J_{\tilde{n}}=\sum_{\alpha=1}^{p-1} n_{\alpha} S_{\alpha},
$$

which is also a complex structure.

Definition 3. The complex structure $J_{\tilde{n}}$ is called the supercomplex structure for the direction $\widetilde{n}$. The set of supercomplex structures is denoted by $\operatorname{Supcom}\left(\mathbb{E}^{n}, \mathbb{E}^{p}\right)$ :

$$
\operatorname{Supcom}\left(\mathbb{E}^{n}, \mathbb{E}^{p}\right)=\left\{J_{\tilde{n}}: \widetilde{n} \in S^{p-2}\right\} .
$$

Here we state some basic facts on supercomplex structures (cf. [21]).

Proposition 5. Let $J$ be a supercomplex structure of a Hurwitz pair $\left(\mathbb{E}^{n}, \mathbb{E}^{p}\right)$. Then there is $R \in \mathcal{O}(n)$ such that

$$
J=R J_{0} R^{-1}, \quad \text { where } J_{0}=\left[\begin{array}{cc}
0 & I_{n / 2} \\
I_{n / 2} & 0
\end{array}\right] .
$$

Proposition 6. (i) $\operatorname{Supcom}\left(\mathbb{E}^{n}, \mathbb{E}^{p}\right)=\left\{J_{0}\right\}$.

(ii) $\operatorname{Supcom}\left(\mathbb{E}^{4}, \mathbb{E}^{4}\right) \cong \mathbb{P}^{1}(\mathbb{C})$. Every parametrization of $\operatorname{Supcom}\left(\mathbb{E}^{4}, \mathbb{E}^{4}\right)$ gives rise to a holomorphic fibre space $\pi: \mathbb{P}^{3}(\mathbb{C})-\mathbb{P}^{1}(\mathbb{C}) \rightarrow \mathbb{P}^{1}(\mathbb{C})$ such that $\pi^{-1}(\widetilde{n})$ is biholomorphic to $\left(\mathbb{R}^{4}, J_{\tilde{n}}\right)$. 
The assertion of (i) is trivial. For the proof of (ii) we refer to [4], p. 39.

Remark 1. The above fibre space is connected with the Penrose transform $\tau: \mathbb{P}^{3}(\mathbb{C}) \rightarrow S^{4}$. Every fibre $\tau^{-1}(p)$ is biholomorphic to $\mathbb{P}^{1}(\mathbb{C})$. Choosing a point $\infty \in S^{4}$, consider the restriction $\tau^{\prime}$ of $\tau$ to $\mathbb{P}^{3}(\mathbb{C})-\tau^{-1}(\infty)$. Then we can obtain $\tau^{\prime}: \mathbb{P}^{3}(\mathbb{C})-\mathbb{P}^{1}(\mathbb{C}) \rightarrow \mathbb{R}^{4}$. We see that $\tau^{\prime}$ maps $\mathbb{C}_{\tilde{n}}^{2}$ to its realization $\mathbb{R}^{4}$. For details we refer to [4], Chap. III.

Supercomplex structures related to Hurwitz triples will be considered in a separate paper.

4. The Hurwitz equation. In this section we introduce a concept of quantization and obtain the Cauchy-Riemann, Fueter, and Dirac equations as the images of quantizations of the Hurwitz mappings.

Let $k\left[y_{1}, \ldots, y_{p}\right]$ denote the algebra of polynomials over $k(=\mathbb{R}$ or $\mathbb{C})$. By $k\left[\partial / \partial x_{1}, \ldots, \partial / \partial x_{q}\right]$ we denote the algebra of differential operators with constant coefficients on $\mathbb{R}^{q}$, where $x_{1}, \ldots, x_{q}$ are the Cartesian coordinates of $\mathbb{R}^{q}$.

Definition 4. Any algebra homomorphism

is called a quantization.

$$
\underline{\chi}: k\left[y_{1}, \ldots, y_{p}\right] \rightarrow k\left[\partial / \partial x_{1}, \ldots, \partial / \partial x_{q}\right]
$$

Let $M_{n}(k)$ denote the algebra of $n \times n$ matrices over $k$. Then $\underline{\chi}$ can be extended in a natural manner to

$$
\chi: M_{n}(k) \otimes k\left[y_{1}, \ldots, y_{p}\right] \rightarrow M_{n}(k) \otimes k\left[\partial / \partial x_{1}, \ldots, \partial / \partial x_{q}\right] .
$$

This is also called a quantization and we use it hereafter.

We consider a Hurwitz pair $\left(\mathbb{E}^{n}, \mathbb{E}^{p}\right)$ with the Hurwitz mapping given by (8). Hence, by (15),

$$
f=y_{1} S_{1}+y_{2} S_{2}+\ldots+y_{p-1} S_{p-1}+y_{p} I_{n} .
$$

We are going to prove that the well-known field equations can be obtained by quantization of Hurwitz mappings.

4.1. Generalized Fueter equations (cf. [22])

Definition 5. The quantization

$$
\chi_{F}: M_{n}(\mathbb{R}) \otimes \mathbb{R}\left[y_{1}, \ldots, y_{p}\right] \rightarrow M_{n}(\mathbb{R}) \otimes \mathbb{R}\left[\partial / \partial x_{1}, \ldots, \partial / \partial x_{p}\right]
$$

defined by

$$
\underline{\chi}_{F}^{(n, p)}: y_{1} \rightarrow \partial / \partial x_{1}, \ldots, y_{p} \rightarrow \partial / \partial x_{p},
$$

is called the quantization of Fueter type. The operator $D_{F}^{(n, p)}=\chi_{F}^{(n, p)}(f)$, where $f$ is the Hurwitz mapping (17), is the generalized Fueter operator of 
the Hurwitz pair $\left(\mathbb{E}^{n}, \mathbb{E}^{p}\right)$, and the corresponding equation $D_{F}^{(n, p)} \psi=0$ the generalized Fueter equation.

Clearly,

$$
D_{F}^{(n, p)}=\sum_{k=1}^{p-1} S_{k}\left(\partial / \partial x_{k}\right)+I_{n}\left(\partial / \partial x_{p}\right) .
$$

We easily get (cf. [19], [20])

Proposition 7. (i) $D_{F}^{(2,2)} \psi=0$ are the Cauchy-Riemann equations. (ii) $D_{F}^{(4,4)} \psi=0$ is the Fueter equation [6].

4.2. Hurwitz equations (cf. [11])

Definition 6. The quantization $\chi_{D}^{(n, p)}: M_{n}(\mathbb{C}) \otimes C\left[y_{1}, \ldots, y_{p}\right] \rightarrow M_{n}(\mathbb{C}) \otimes \mathbb{C}\left[\partial / \partial x_{1}, \ldots, \partial / \partial x_{p-1}, \partial / \partial t\right]$, defined by

$$
\underline{\chi}_{D}^{(n, p)}: y_{1} \rightarrow \partial / \partial x_{1}, \ldots, y_{p-1} \rightarrow \partial / \partial x_{p-1}, y_{p} \rightarrow-i \partial / \partial t,
$$

is called the quantization of Dirac type (or time-separation quantization). The operator $D^{(n, p)}=\chi_{D}^{(n, p)}(f)$ is the time-dependent Hurwitz operator of the Hurwitz pair $\left(\mathbb{E}^{n}, \mathbb{E}^{p}\right)$. The equation $D^{(n, p)} \psi=0$ is the time-dependent Hurwitz equation.

The latter can be written as

$$
i \partial \psi / \partial t=\sum_{j=1}^{p-1} S_{j} \partial \psi / \partial x_{j} .
$$

Then we get (cf. [11])

Proposition 8. $D^{(4,4)} \psi=0$ is the non-relativistic massless Dirac equation.

Remark 2. Operating with $D^{(n, p)}$ and

$$
D^{(n, p) *}=i(\partial / \partial t)+\sum_{k=1}^{p-1} S_{k}\left(\partial / \partial x_{k}\right),
$$

we obtain the so-called Klein-Gordon operator

$$
D^{(n, p)} D^{(n, p) *}=\left(\partial^{2} / \partial t^{2}-\Delta_{p-1}\right) I_{n},
$$

where $\Delta_{p-1}$ is the usual Laplacian on $\mathbb{R}^{p-1}$.

4.3. The mass-producing quantization (cf. [12] and [7]; the introduction of mass by use of spontaneous symmetry breaking of the gauge structure of the Hurwitz operator has been suggested by Dr. S. Kanemaki). In order to get mass in physics, we consider the following quantization: 
Definition 7. The quantization

$$
\chi_{m}^{(n, p)}: M_{n}(\mathbb{C}) \otimes \mathbb{C}\left[y_{1}, \ldots, y_{p}\right] \rightarrow M_{n}(\mathbb{C}) \otimes \mathbb{C}\left[\partial / \partial x_{1}, \ldots, \partial / \partial x_{p-1}\right]
$$

defined by

$$
\underline{\chi}_{m}^{(n, p)}: y_{1} \rightarrow \partial / \partial x_{1}, \ldots, y_{p-1} \rightarrow \partial / \partial x_{p-1}, y_{p} \rightarrow m \cdot 1,
$$

where $m$ is a non-negative constant and 1 is the identity operator, is called the mass-producing quantization. The operator $\mathcal{H}_{m}^{(n, p)}=\chi_{m}^{(n, p)}(f)$ is the Hurwitz operator of the Hurwitz pair $\left(\mathbb{E}^{n}, \mathbb{E}^{p}\right)$ with mass $m$. In particular, when we choose $m=0$, we call it the Hurwitz operator and denote by $\mathcal{H}^{(n, p)}$. The equation $\mathcal{H}^{(n, p)} \psi=0$ is the Hurwitz equation.

The Hurwitz operator can be written as

$$
\mathcal{H}^{(n, p)}=\sum_{k=1}^{p-1} i \gamma_{k} \partial / \partial x_{k}
$$

We now investigate its (formal) self-adjointness. Consider the linear space $\Gamma_{0}\left(\mathbb{R}^{p-1}, \mathbb{C}^{n}\right)$ of $n$-component $C^{\infty}$ functions with compact supports. Then

$$
\mathcal{H}^{(n, p)}: \Gamma_{0}\left(\mathbb{R}^{p-1}, \mathbb{C}^{n}\right) \rightarrow \Gamma_{0}\left(\mathbb{R}^{p-1}, \mathbb{C}^{n}\right) .
$$

We introduce an inner product by

$$
(\phi, \psi)=\int_{\mathbb{R}^{p-1}} \phi^{*} \psi d v \quad \text { for } \phi, \psi \in \Gamma_{0}\left(\mathbb{R}^{p-1}, \mathbb{C}^{n}\right) .
$$

Then the formal adjoint operator $\mathcal{H}^{(n, p) *}$ of $\mathcal{H}^{(n, p)}$ is given by

$$
\mathcal{H}^{(n, p) *}=\sum_{k=1}^{p-1} i \gamma_{k}^{*} \partial / \partial x_{k}
$$

Remark 3. The operator $\mathcal{H}^{(n, p)}$ is formally self-adjoint if and only if $\gamma_{k}^{*}=\gamma_{k}, k=1, \ldots, p-1$.

In analogy to [11] and [12], it seems important to give a characterization of pre-Hurwitz pairs in terms of Hurwitz equations:

THEOREM 1. The following statements are equivalent:

(i) $\left(\mathbb{E}^{n}, \mathbb{E}^{p}\right)$ is a pre-Hurwitz pair.

(ii) There exist purely imaginary $n \times n$ matrices $\gamma_{1}, \ldots, \gamma_{p-1}$ which satisfy (6) and (7).

(iii) There exist purely imaginary $n \times n$ matrices $\gamma_{1}, \ldots, \gamma_{p-1}$ such that $\mathcal{H}$ defined by $(20)$ is a self-adjoint operator satisfying

$$
\mathcal{H}^{2}=-\Delta_{p-1} I_{n}
$$

Proof. (i) $\Leftrightarrow($ ii) is a direct consequence of the considerations in Section 1 . 
(ii) $\Rightarrow$ (iii). For $\gamma_{1}, \ldots, \gamma_{p-1}$ as in (ii), the self-adjointness of the operator $\mathcal{H}$ given by (20) follows from (7). We can also see that (22) follows from (6).

(iii) $\Rightarrow$ (ii). Since $\mathcal{H}$ is self-adjoint, we have $\gamma_{k}^{*}=\gamma_{k}, k=1, \ldots, p-1$. Since $\gamma_{k}$ are all purely imaginary, the relations (7) hold, and (22) implies (6), as desired.

Remark 4. The Hurwitz conditions (1) can be restated in terms of the Hurwitz operator $\mathcal{H}^{(n, p)}$ as follows:

$$
\left(-\Delta_{p-1} I_{n} \psi, \psi\right)=\left\|\mathcal{H}^{(n, p)} \psi\right\|^{2} \quad \text { for } \psi \in \Gamma_{0}\left(\mathbb{R}^{p-1}, \mathbb{C}\right),
$$

where \|\| is defined by the inner product (21).

5. The Neveu-Schwarz model. In this section we shall concentrate on the complex version of the Hurwitz pairs $\left(\mathbb{E}^{4}, \mathbb{E}^{3}\right)$, and obtain the equations of the four-dimensional elliptic Neveu-Schwarz model in superstring theory [31].

First we have to treat the Hurwitz condition in complex form. This leads to the concept of hermitian Hurwitz pairs. Detailed studies are given in [14] and [17], but without emphasizing their relation to the Neveu-Schwarz model.

We identify the first element $\mathbb{E}^{4}$ of $\left(\mathbb{E}^{4}, \mathbb{E}^{4}\right)$ with the hermitian space $\mathbb{E}_{\mathbb{C}}^{2}$ in a natural manner and consider the Hurwitz mapping $f_{\mathbb{C}}: \mathbb{E}_{\mathbb{C}}^{2} \times \mathbb{E}^{4} \rightarrow \mathbb{E}_{\mathbb{C}}^{2}$. It is called the complex form of the Hurwitz mapping $f$. We are going to write it down explicitly. First we notice that the Hurwitz mapping of $\left(\mathbb{E}^{4}, \mathbb{E}^{4}\right)$ can be written as

$$
f(x, y)=\left(-i y_{1} \gamma_{1}-i y_{2} \gamma_{2}-i y_{3} \gamma_{3}+y_{4} I_{4}\right) x,
$$

where

$$
\gamma_{1}=i\left[\begin{array}{cc}
0 & \sigma_{1} \\
-\sigma_{1} & 0
\end{array}\right], \quad \gamma_{2}=\left[\begin{array}{cc}
\sigma_{2} & 0 \\
0 & \sigma_{2}
\end{array}\right], \quad \gamma_{3}=i\left[\begin{array}{cc}
0 & \sigma_{3} \\
-\sigma_{3} & 0
\end{array}\right] .
$$

Here $\sigma_{1}, \sigma_{2}, \sigma_{3}$ are the well-known Pauli matrices

$$
\sigma_{1}=\left[\begin{array}{ll}
0 & 1 \\
1 & 0
\end{array}\right], \quad \sigma_{2}=\left[\begin{array}{cc}
0 & -i \\
i & 0
\end{array}\right], \quad \sigma_{3}=\left[\begin{array}{cc}
1 & 0 \\
0 & -1
\end{array}\right] .
$$

Then we see that the complex form $f_{\mathbb{C}}: \mathbb{E}_{\mathbb{C}}^{2} \times \mathbb{E}^{4} \rightarrow \mathbb{E}_{\mathbb{C}}^{2}$ of the Hurwitz mapping (23) reads

$$
f_{\mathbb{C}}=\left(i y_{1} \sigma_{1}+i y_{2} \sigma_{2}+i y_{3} \sigma_{3}+y_{4} I_{2}\right) x .
$$

By Proposition 4 we conclude that $\left(\mathbb{E}^{4}, \mathbb{E}^{3}\right)$ is also a Hurwitz pair. The Hurwitz mapping and its complex form are

$$
f(x, y)=\left(-i y_{1} \gamma_{1}-i y_{2} \gamma_{2}+y_{3} I_{4}\right) x
$$

and 


$$
f_{\mathbb{C}}(x, y)=\left(i y_{1} \sigma_{1}+i y_{2} \sigma_{2}+y_{3} I_{2}\right) x
$$

respectively. We obtain

THEOREM 2. The Hurwitz mapping (24) in its complex form (25) gives rise to the system of Hurwitz equations

$$
\sigma_{1}(\partial / \partial \sigma) \psi+i \sigma_{2}(\partial / \partial \tau) \psi=0, \quad\left(\partial^{2} / \partial \sigma^{2}\right) \phi-\left(\partial^{2} / \partial \tau^{2}\right) \phi=0,
$$

which is equivalent to the system

$$
\begin{aligned}
& (\partial / \partial \sigma)\left(\lambda^{1}\right)^{k}+(\partial / \partial \tau)\left(\lambda^{1}\right)^{k}=0, \quad(\partial / \partial \sigma)\left(\lambda^{2}\right)^{k}-(\partial / \partial \tau)\left(\lambda^{2}\right)^{k}=0, \\
& \left(\partial^{2} / \partial \sigma^{2}\right) x^{k}-\left(\partial^{2} / \partial \tau^{2}\right) x^{k}=0,
\end{aligned}
$$

describing, in the notation of [31], the four-dimensional Neveu-Schwarz sectors, by the substitution

$$
\lambda=A \psi, \quad A=\left[\begin{array}{ll}
0 & 1 \\
1 & 0
\end{array}\right], \lambda=\left[\begin{array}{c}
\lambda_{1} \\
\lambda_{2}
\end{array}\right] .
$$

Pro of. Consider the mass-producing quantization

$$
\chi_{0}: M_{2}(\mathbb{C}) \otimes \mathbb{C}\left[y_{1}, y_{2}, y_{3}\right] \rightarrow M_{2}(\mathbb{C}) \otimes \mathbb{C}[\partial / \partial \sigma, \partial / \partial \tau]
$$

defined, according to (19), by

$$
\underline{\chi}_{0}: y_{1} \rightarrow-i \partial / \partial \sigma, y_{2} \rightarrow \partial / \partial \tau, y_{3} \rightarrow m \cdot 1 \equiv 0 \cdot 1 .
$$

This may be regarded as the complex version of the mass-producing quantization of Section 4.3, given in (19). Then $\mathcal{H}_{\mathbb{C}}^{(4,3)}=\chi_{0}\left(f_{\mathbb{C}}\right)$ becomes

$$
\mathcal{H}_{\mathbb{C}}^{(4,3)}=\sigma_{1}(\partial / \partial \sigma)+i \sigma_{2}(\partial / \partial \tau)
$$

The corresponding Klein-Gordon operator (18) is

$$
\left(\partial^{2} / \partial \sigma^{2}\right)-\left(\partial^{2} / \partial \tau^{2}\right)
$$

and (26) are the corresponding Hurwitz equations.

We shall prove that the system (26), by a suitable substitution, is equivalent to the system (27), as desired. Consider the lagrangian of the corresponding Neveu-Schwarz model:

$$
\mathcal{L}=\left(-1 / 2 \alpha^{\prime}\right) \int d \sigma d \tau \sum_{\alpha=1}^{2} \sum_{k=1}^{2}\left(\partial_{\alpha} x^{k} \partial^{\alpha} x^{k}+i \lambda^{-k} \rho^{\alpha} \partial_{\alpha} \lambda^{k}\right),
$$

where

$$
\rho^{1}=\left[\begin{array}{cc}
0 & -i \\
i & 0
\end{array}\right], \quad \rho^{2}=\left[\begin{array}{cc}
0 & i \\
i & 0
\end{array}\right],
$$

and $\lambda^{k}, k=1,2$, are two-component spinors; see [31], p. 240, formula (2.32). The Euler-Lagrange equations of the lagrangian are (27) and we can easily see that the system (27) is, by the substitution (28), equivalent to (26), as desired. 
The four-dimensional Neveu-Schwarz model is a toy model which, in the language of the generalized Hurwitz condition (13), is connected with the solvability of the algebraic equation

$$
(a f)_{1}^{2}+(a f)_{2}^{2}+(a f)_{3}^{2}+(a f)_{4}^{2}=\left(a_{1}^{2}+a_{2}^{2}+a_{3}^{2}\right)\left(f_{1}^{2}+f_{2}^{2}+f_{3}^{2}+f_{4}^{2}\right),
$$

where

$$
(a f)_{A}=\sum_{j=1}^{4} \sum_{\alpha=1}^{3} c_{A}^{j \alpha} a_{\alpha} f_{j}, \quad c_{A}^{j \alpha} \in \mathbb{R}, A=1, \ldots, 4 .
$$

The general Neveu-Schwarz model [31], [8] (see, in particular, p. 202) includes a study of the interrelation between the bosonic or Neveu-Schwarz sectors, i.e. sectors with half-integrally moded world-sheet spinors, and the fermionic or Ramond sectors, i.e. sectors with integrally moded world-sheet spinors.

The bosonic states are connected with the real dimension 26 , the fermionic states with the dimension 10 or 11 (ten-dimensional Stiefel manifolds embedded in $\mathbb{R}^{11}$; cf. [21]). In the language of the generalized Hurwitz condition (14), the model is connected with the solvability of the algebraic equation

$$
\begin{aligned}
(a f)_{1}^{2}+\ldots+(a f)_{N_{0}}^{2}- & (a f)_{N_{0+1}}^{2}-\ldots-(a f)_{N}^{2} \\
& =\left(a_{1}^{2}+\ldots+a_{1}^{2}-a_{11}^{2}\right)\left(f_{1}^{2}+\ldots+f_{1}^{2}-f_{11}^{2}\right),
\end{aligned}
$$

where

$$
(a f)_{A}=\sum_{j=1}^{11} \sum_{\alpha=1}^{11} c_{A}^{j \alpha} a_{\alpha} f_{j}, \quad c_{A}^{j \alpha} \in \mathbb{R}, A=1, \ldots, N .
$$

It can be proved that the minimal value of $N$ is 26 [2], as required in physics, and a counterpart of Theorem 1 for the bosonic and fermionic sectors has to involve Hurwitz triples $(S, V, W)$ with a Hurwitz mapping $S \times V \rightarrow W$ instead of Hurwitz pairs $(S, V)$ with a Hurwitz mapping (multiplication) $S \times V \rightarrow V$. The problem requires further study.

6. Isospectral deformations of the Hurwitz operator of $\left(\mathbb{E}^{2}, \mathbb{E}^{2}\right)$. In [3], we gave isospectral deformations of the Hurwitz operator in the case of $n=p=2$ and obtained the KdV (=Korteweg-de Vries) and the modified $\mathrm{KdV}$ equations. Unfortunately, other important equations fell out of our considerations. In this section we shall give a full description of isospectral deformations of the Hurwitz operator $\mathcal{H}\left(=\mathcal{H}^{(2,2)}\right)$. Isospectral deformations of Hurwitz operators in more general cases are important in connection with gauge fields and will be discussed in a forthcoming paper. Firstly, we are going to prove that isospectral deformations of $\widehat{\mathcal{H}}(=i \mathcal{H})$ are equivalent to those treated by Ablowitz et al. [1]. Hence we may say that the 
known soliton equations are obtained by use of isospectral deformations from our Hurwitz operator. Secondly, we shall apply the 2-component K.P. (=Kadomtsev-Petviashvili) theory, due to M. and Y. Sato [28], to $\widehat{\mathcal{H}}$ and discuss soliton equations in our case.

We set

$$
\widehat{\mathcal{H}}=\sigma D, \quad \text { where } \quad \sigma=\left[\begin{array}{cc}
0 & -i \\
i & 0
\end{array}\right] \text { and } D=d / d x,
$$

and consider an isospectral deformation of $\widehat{\mathcal{H}}$ with a deformation parameter $t$ whose generator is given by a differential operator $B$ :

$$
\begin{aligned}
L \psi & =\lambda \psi, \quad \text { where } \quad L=\widehat{\mathcal{H}}+U, \\
\psi_{t} & =B \psi,
\end{aligned}
$$

$\lambda$ is an isospectral parameter, and $U(=U(x, t))$ is a matrix-valued function. We are going to show that (29) can be transformed to the isospectral deformation introduced by Ablowitz et al. [18]. Put

$$
A=\left[\begin{array}{cc}
-i & -1 \\
1 & i
\end{array}\right]
$$

and consider the matrix $A^{-1} \widehat{\mathcal{H}} A$. We see that it is equal to

$$
K D, \quad \text { where } \quad K=\left[\begin{array}{cc}
1 & 0 \\
0 & -1
\end{array}\right] \text {, }
$$

which is nothing but the operator introduced by Ablowitz. Thus we can transform his results to our case. We have (cf. [18] and [32])

Proposition 9. By isospectral deformations (29), we can obtain the $K d V$, sine-Gordon, and nonlinear Schrödinger equations. More exactly, if we set

$$
U=\frac{1}{2}\left[\begin{array}{cc}
-(r+q) i & -r+q \\
-r+q & (r+q) i
\end{array}\right]
$$

where $r$ and $q$ are functions of $x$ and $t$, then we obtain:

(i) the modified $K d V$ equation $r_{t}+6 r^{2} r_{x}+r_{x x x}=0$ for $r=q$ real and $B$ a differential operator of the third order,

(ii) the nonlinear Schrödinger equation $i_{t}+r_{x x}+2|r|^{2}=0$ for $r=q^{*}$, the complex conjugate of $q$, and $B$ a differential operator of the second order,

(iii) the sine-Gordon equation $r_{t x}=\sin r$ for $r=q$ real and $B$ a pseudodifferential operator of order -1 .

Next we define the K.-P. system for the operator $\mathcal{H}$. Following [30], we introduce a pseudo-differential operator

$$
P=P_{0}+P_{1} D^{-1}+\ldots+P_{n} D^{-n}+\ldots,
$$


where $P_{n}=P_{n}(x)$ is a matrix-valued function and $P_{0}$ is a constant invertible matrix. We set

$$
L=P \widehat{\mathcal{H}} P^{-1} \quad \text { and } \quad L_{n}=P \sigma D^{n} P^{-1} .
$$

Then we have the decomposition

$$
L_{n}=\left(L_{n}\right)_{+}+\left(L_{n}\right)_{-}
$$

with

$$
\begin{aligned}
& \left(L_{n}\right)_{+}=B_{n}^{(n)} D^{n}+B_{n-1}^{(n)} D^{n-1}+\ldots+B_{0}^{(n)}, \\
& \left(L_{n}\right)_{-}=B_{-1}^{(n)} D^{-1}+B_{-2}^{(n)} D^{-2}+\ldots
\end{aligned}
$$

We notice that $B_{n}^{(n)}=P_{0} \sigma D^{n} P_{0}^{-1}$. Here we introduce an infinite number of time parameters $t=\left(t_{1}, t_{2}, \ldots\right)$ and consider $P(=P(t))$ as depending on $t$. We introduce

Definition 8. The equation

$$
\partial L / \partial t_{n}=\left[\left(L_{n}\right)_{+}, L\right], \quad n=1,2, \ldots,
$$

is called the K.-P. (=Kadomtsev-Petviashvili) system of the Hurwitz pair $\left(\mathbb{E}^{2}, \mathbb{E}^{2}\right)$.

Following the discussions in [26], we can prove that every solution of (31) can be obtained from a solution of the linear equation

$$
\partial U / \partial t_{n}=\sigma D^{n} U, \quad n=1,2, \ldots
$$

We get special solutions of (31):

Proposition 10. Any K.-P. system (31) can be reduced to the K. $-P$. system for $K D$, where $A^{-1}$ is taken for $P_{0}$, and $A$ is given by (30). Hence, the nonlinear Schrödinger equation and the modified $K d V$ equation can be obtained in the cases of $n=2$ and $n=3$, respectively.

Proof. We choose $P_{0}=A^{-1}$. For a solution $P$ of $(31)$, we set

$$
\begin{aligned}
& P=P^{\prime} A^{-1}, \quad P^{\prime}=I_{2}+P_{1}^{\prime} D^{-1}+P_{2}^{\prime \prime} D^{-2}+\ldots, \\
& L^{\prime}=P^{\prime} K D P^{\prime-1}, \quad L_{n}^{\prime}=P^{\prime} K D^{n} P^{\prime-1} .
\end{aligned}
$$

Then we see that $L^{\prime}$ satisfies

$$
\partial L^{\prime} / \partial t_{n}=\left[\left(L_{n}^{\prime}\right)_{+}, L^{\prime}\right], \quad n=1,2, \ldots,
$$

which is the K.-P. system for $K D$. The latter part of the assertion is well known (calculating the integrability conditions in the case of $n=1,2$, we can obtain the so-called nonlinear Schrödinger equation; private communication by Dr. Jimbo).

By summarizing the above considerations we obtain the following 
THEOREM 3. Isospectral deformations of the Hurwitz operator $\mathcal{H}$ of $\left(\mathbb{E}^{2}, \mathbb{E}^{2}\right)$ give rise to the $K d V$, modified $K d V$, sine-Gordon, and nonlinear Schrödinger equations. The K.-P. system for $\mathcal{H}$ can be defined by (31) and its linearization is given by (32). In the cases of $n=2,3$ this equation gives the nonlinear Schrödinger and modified $K d V$ equations, respectively.

7. Supercomplex structure of a Hilbert space and reduction solutions of the K.-P. system. In this section we generalize the concept of supercomplex structure to a Hilbert space and establish a correspodence between supercomplex structures and reduction solutions of the original K.-P. system. We know that reduction solutions of the K.-P. system give solutions of the KdV and other soliton equations [5]. On the other hand, we know that the Virasoro algebra is used to describe soliton equations [29]. Taking into account the fact that the Virasoro algebra can be obtained from the group of biholomorphic mappings of $\mathbb{C}^{*}$, we may say that our result gives one of the explanations why the Virasoro algebra is used in the theory of soliton equations.

We begin with generalizing the concept of a pre-Hurwitz pair to a Hilbert space. By $\mathbb{H}$ we denote a separable Hilbert space over $\mathbb{R}$. We give the following definition (cf. [21], Section 3):

Definition 9. A pair $\left(\mathbb{H}, \mathbb{E}^{p}\right)$ is called a pre-Hurwitz pair if there exists a bilinear mapping $f: \mathbb{H} \times \mathbb{E}^{p} \rightarrow \mathbb{H}$ satisfying

$$
\|f(x, y)\|=\|x\|\|y\| \quad \text { for any } x \in \mathbb{H} \text { and } y \in \mathbb{E}^{p},
$$

where $\|x\|,\|y\|$ denote the norms of $\mathbb{H}$ and $\mathbb{E}^{p}$, respectively. A pre-Hurwitz pair is called decomposable if $\mathbb{H}$ has a decomposition

$$
\mathbb{H}=\bigoplus_{k=1}^{\infty} \mathbb{E}_{k}^{n}, \quad \text { where }\left(\mathbb{E}_{k}^{n}, \mathbb{E}^{p}\right) \text { is a Hurwitz pair . }
$$

In the following we consider only decomposable pre-Hurwitz pairs.

Definition 10. Choosing supercomplex structures $J_{k}$ in $\mathbb{E}_{k}^{n}$, we define a (decomposable) supercomplex structure $J$ on $\mathbb{H}$ by

$$
J=\bigoplus_{k=1}^{\infty} J_{k}
$$

Then we can prove the following

THEOREM 4. There exists a correspondence between the set of decomposable supercomplex structures and the set of $\mathfrak{s} \mathfrak{o}(p-1)$-reduction solutions of the original version of the K.-P. system.

Before proceeding to the proof of Theorem 4, we recall basic facts on reduction solutions of the K.-P. system [5]. The original K.-P. system is 
given in the following manner: We set

$L=W D W^{-1}, \quad$ where $\quad D=d / d x$ and $W=1+u_{1} D^{-1}+u_{2} D^{-2}+\ldots$

Here $u_{1}, u_{2}, \ldots$ are functions of $x$ and $t, t=\left(t_{1}, t_{2}, \ldots\right)$. We call the equation

$$
\partial L / \partial t_{n}=\left[\left(L^{n}\right)_{+}, L\right]
$$

the (original) K.-P. system [30]. A solution of the K.-P. system is called an $\ell$-reduction solution if

$$
\left(L^{\ell}\right)_{+}=L^{\ell}
$$

with some integer $\ell$. We know that 2-reduction (or 5-reduction) solutions give rise to those of the $\mathrm{KdV}$ (resp. Boussinesq) equations. It is well known that $\ell$-reduction solutions can be characterized in terms of the so-called Kac-Moody Lie algebra, more exactly the Lie algebra $A_{\ell-1}^{(1)}$ [5]. An element $\xi \in A_{\ell-1}^{(1)}$ can be expressed as

$$
\xi=\sum_{k=-\infty}^{\infty} X^{(k)} \lambda^{k},
$$

where $X^{(k)} \in \mathfrak{s l}(\ell, \mathbb{R})$ and $\lambda$ is a parameter. In a similar manner we can define $\mathcal{G}$-reduction solutions. We choose a Lie subalgebra $\mathcal{G}$ of $\mathfrak{s l}(\ell, \mathbb{R})$ and define $\xi$ by $(34)$ with $X^{(k)} \in \mathcal{G}(k \in \mathbb{Z})$. The corresponding solutions of the K.-P. system are called $\mathcal{G}$-reduction solutions.

Proof of Theorem 4. Let $\xi$ be an $\mathfrak{s o}(p-1)$-reduction solution. Then $\xi$ has the form (34). Hence we have a sequence of infinite elements $\left\{X^{(k)}\right\}_{k \in \mathbb{Z}}, X^{(k)} \in \mathfrak{s o}(p-1, \mathbb{R})$. We set $g_{k}=\exp \left(X^{(k)}\right)(\in \operatorname{SO}(p-1, \mathbb{R}))$. By use of the identification (see (16))

$$
\tau^{-1}: \operatorname{Supcom}\left(\mathbb{E}^{n}, \mathbb{E}^{p}\right) \rightarrow \mathrm{SO}(p-1, \mathbb{R}) / \mathrm{SO}(p-2, \mathbb{R})
$$

we get the elements $J_{k}=\tau\left(g_{k}\right), k \in \mathbb{Z}$, so we obtain a supercomplex structure of $\mathbb{H}$.

Conversely, we choose a supercomplex structure $J$ of $\mathbb{H}$. Then we have a sequence of infinite supercomplex structures $J_{k}$ of the form (33). By (35) we have elements $g_{k}$ of $\mathrm{SO}(p-1, \mathbb{R})$ satisfying $J_{k}=\tau\left(g_{k}\right)$. Then we arrive at the elements $X^{(k)}$ of $\mathfrak{s o}(p-1, \mathbb{R}), k \in \mathbb{Z}$. Finally, we obtain an $\mathfrak{s o}(p-1, \mathbb{R})$ reduction solution of the form $(34)$, and this completes the proof.

The above construction and Theorem 4 are also valid in a more general context, namely if we take a pseudo-euclidean Hurwitz pair $\left(\mathbb{E}_{k}^{(n, \ell)}, \mathbb{E}^{(p, r)}\right)$, instead of the Hurwitz pair $\left(\mathbb{E}_{k}^{n}, \mathbb{E}^{p}\right)$, provided that $n$ and $\ell$ are even integers, where $\eta_{\alpha \alpha}=1$ for $1 \leq j \leq n, \eta_{\alpha \alpha}=-1$ for $n+1 \leq j \leq n+\ell[17]$.

Theorems 3 and 4 are connected with the Hurwitz operators and the corresponding K.-P. system having, in general, soliton solutions. An important example is provided by a system of electrons, bounded by a surface. In that 
case the flux of the electromagnetic field, manifesting itself as a deformation of the space outside the object, determines in a natural way the demagnetizing fields existing in the region over the surface. The occurrence of those fields, experimentally measurable, enables us to determine the boundary conditions for solutions describing the electron density distribution inside the system. In other words, the boundary conditions can be expressed via deformation of the metric in near-surface regions [15].

In particular, the motion of a ferroelectric wall with electromechanical couplings in a ferroelectric crystal is described by the double sine-Gordon equation

$$
u_{x t}=\sin u-\gamma \sin 2 u,
$$

where $\gamma$ is a real constant (cf. Proposition 9). One of the solutions of that equation is stable and gives an example of surface solitons if the maximum is attained on the surface of the crystal.

Another example is given by the class of quasiconformal mappings in the plane (or, more generally, on a Riemann surface), which will be considered in the next section because of the close relationship between the parametrical method (shown in [12] as a particular case of the method of isospectral deformations) and the boundary behaviour of quasiconformal mappings.

8. Generalized Hurwitz pairs and quasiconformal mappings. In this section we generalize the concept of a Hurwitz pair to vector spaces with more general metrics and show that such a pair induces a quasiconformal mapping.

For a real-valued function $\sigma$ on $\mathbb{R}^{n}$ with $\sigma(x) \neq 0$ for any $x \in \mathbb{R}^{n}$, consider the metric \|\|$_{\sigma}=\sigma^{2}\|\|$, where \|\| is the usual Euclidean metric on $\mathbb{R}^{n}$. The space $\mathbb{R}^{n}$ endowed with the metric \|\|$_{\sigma}$ is denoted by $E_{\sigma}^{n}$.

We now choose functions $\sigma^{\prime}$ and $\sigma$ as above on $\mathbb{R}^{n}$ and $\mathbb{R}^{p}$, respectively, and consider the pair $\left(\mathbb{E}_{\sigma^{\prime}}^{n}, \mathbb{E}_{\sigma}^{p}\right)$ and the Hurwitz condition for this pair:

Definition 11. If a mapping $f: \mathbb{E}_{\sigma^{\prime}}^{n} \times \mathbb{E}_{\sigma}^{p} \rightarrow \mathbb{E}_{\sigma^{\prime}}^{n}$ satisfies

$$
\|f(x, y)\|_{\sigma^{\prime}}=\|x\|_{\sigma^{\prime}}\|y\|_{\sigma} \text { for any } x \in \mathbb{E}_{\sigma^{\prime}}^{n} \text { and } y \in \mathbb{E}_{\sigma}^{p},
$$

we say that $f$ satisfies the generalized Hurwitz condition. A pair $\left(\mathbb{E}_{\sigma^{\prime}}^{n}, \mathbb{E}_{\sigma}^{p}\right)$ satisfying this condition is called a generalized pre-Hurwitz pair. Moreover, if $f$ satisfies the irreducibility condition (see (3)), the pair is called a generalized Hurwitz pair.

Remark 5. The condition (36) can be written as

$$
\|f(x, y)\|=\|x\|\|y\|_{\sigma} .
$$

Hence only $\sigma$ is essential in (36), and not $\sigma^{\prime}$. Therefore, in the following we restrict our considerations to generalized Hurwitz pairs $\left(\mathbb{E}^{n}, \mathbb{E}_{\sigma}^{p}\right)$. 
We derive a counterpart of the condition (5) for a generalized Hurwitz pair. We set

$$
f_{\sigma}(x, y)=\sigma^{-2} f(x, y) \text {. }
$$

Then we can easily see that $f_{\sigma}(x, y)$ satisfies $(1)$. Next we show that $f_{\sigma}$ is a bilinear mapping. In fact, set

$$
\phi_{y_{0}}^{(\sigma)}(x):=\left\|y_{0}\right\|^{-1} f\left(x, y_{0}\right) \quad \text { for } y_{0}(\neq 0) \in \mathbb{R}^{p} \text {. }
$$

This is an isometry and hence it is linear with respect to $x$. Therefore $f(x, y)$ is linear with respect to $x$. In a similar manner, we see that it is also linear with respect to $y$. Hence, by Proposition 3 , we can obtain matrices $C_{\alpha}$, $\alpha=1, \ldots, p$, which satisfy the conditions (4) and (5) with respect to $f_{\sigma}$. We set

$$
C_{\alpha}(\sigma)=\sigma C_{\alpha}, \quad \alpha=1, \ldots, p-1, \quad C_{p}(\sigma)=C_{p} .
$$

Then we can see that

$$
\begin{aligned}
& C_{\alpha}^{T}(\sigma) C_{\beta}(\sigma)+C_{\beta}^{T}(\sigma) C_{\alpha}(\sigma)=2 \sigma^{2} \delta_{\alpha \beta} I_{n}, \quad \alpha, \beta=1, \ldots, p-1, \\
& C_{\alpha}^{T}(\sigma) C_{p}(\sigma)+C_{p}^{T}(\sigma) C_{\alpha}(\sigma)=0, \quad C_{p}^{T}(\sigma) C_{p}(\sigma)=I_{n}, \quad \alpha=1, \ldots, p-1 .
\end{aligned}
$$

Now we define $\gamma_{\alpha}(\sigma), \alpha=1, \ldots, p-1$, by

$$
C_{\alpha}(\sigma)=i C_{p}(\sigma) \gamma_{\alpha}(\sigma) \text {. }
$$

Then, as in Section 1, we have

$$
\begin{aligned}
& \gamma_{\alpha}(\sigma) \gamma_{\beta}(\sigma)+\gamma_{\beta}(\sigma) \gamma_{\alpha}(\sigma)=2 \sigma^{2} \delta_{\alpha \beta} I_{n}, \\
& \gamma_{\alpha}^{T}(\sigma)=-\gamma_{\alpha}(\sigma), \quad \operatorname{re} \gamma_{\alpha}(\sigma)=0,
\end{aligned} \quad \alpha, \beta=1, \ldots, p-1 .
$$

We define the generalized Fueter equation by

$$
\widehat{\mathcal{H}}_{n, p}(\sigma) \psi=0, \quad \text { where } \quad \widehat{\mathcal{H}}_{n, p}(\sigma)=-\sum_{k=1}^{p-1} i \gamma_{k}\left(\partial / \partial x_{k}\right)+I_{n}\left(\partial / \partial x_{p}\right) .
$$

Hereafter we restrict ourselves to the case $n=p=2$. We choose a nonvanishing real-valued Lebesgue-measurable function $\sigma$ on the two-dimensional sphere $S^{2}$ with coordinates $\left(x_{1}, x_{2}\right)$, satisfying

$$
\|(1-\sigma) /(1+\sigma)\|_{\infty}<1,
$$

and consider the related metric

$$
d s^{2}=\sigma^{2}\left(d x_{1}^{2}+d x_{2}^{2}\right)
$$

(cf. [19], [27], [28]), the generalized Hurwitz pair $\left(\mathbb{E}^{2}, \mathbb{E}_{\sigma}^{2}\right)$ with

(38) $\quad \gamma(\sigma)=\gamma(\sigma, \theta):=\frac{1}{\cos ^{2} \theta+\sigma^{2} \sin ^{2} \theta}$

$$
\times\left[\begin{array}{cc}
-i\left(\sigma^{2}-1\right) \cos \theta \sin \theta & -i \sigma \\
i \sigma & -i\left(\sigma^{2}-1\right) \cos \theta \sin \theta
\end{array}\right]
$$


and an arbitrary real-valued Lebesgue-measurable function $\theta$. The corresponding Fueter equation is

$$
\left[I_{2}\left(\partial / \partial x_{2}\right)-i \gamma(\sigma, \theta)\left(\partial / \partial x_{1}\right)\right] \psi=0 \quad \text { (almost everywhere) } .
$$

Then we can prove the following generalization of Theorem 3 in [14]:

TheOREM 5. Deformations of the metric (37) give rise to a generalized Fueter equation (39) with $\gamma$ given by (38), which corresponds to the generalized Hurwitz pair $\left(\mathbb{E}^{2}, \mathbb{E}_{\sigma}^{2}\right)$. The equation (39) with $\gamma$ given by (38) is equivalent to the Beltrami equation

$$
\partial_{\bar{z}} f=\mu \partial_{z} f \quad \text { (almost everywhere) }
$$

of a quasiconformal mapping $f$, by the substitution

$$
\begin{gathered}
\psi=\left[\begin{array}{l}
\psi_{1} \\
\psi_{2}
\end{array}\right], \quad f=\psi_{1}+i \psi_{2}, \\
z=x_{2}+i x_{1}, \quad \mu=\frac{\sigma-1}{\sigma+1} e^{2 i \theta} .
\end{gathered}
$$

Proof. The equation (39) with $\gamma$ given by (38) is equivalent to the system

$$
\begin{aligned}
& \left(\cos ^{2} \theta+\sigma^{2} \sin ^{2} \theta\right) \frac{\partial}{\partial x_{2}} \psi_{1}=\left(\sigma^{2}-1\right) \cos \theta \sin \theta \frac{\partial}{\partial x_{1}} \psi_{1}+\sigma \frac{\partial}{\partial x_{1}} \psi_{2} \\
& \left(\cos ^{2} \theta+\sigma^{2} \sin ^{2} \theta\right) \frac{\partial}{\partial x_{2}} \psi_{2}=-\sigma \frac{\partial}{\partial x_{1}} \psi_{2}+\left(\sigma^{2}-1\right) \cos \theta \sin \theta \frac{\partial}{\partial x_{1}} \psi_{2}
\end{aligned}
$$

a.e. on $S^{2}$.

On the other hand, we observe that (40) with $\mu, f$ and $z$ as in (41) can be written a.e. on $S^{2}$ in the form

$$
\begin{aligned}
& (\sigma+1) \cos \theta\left(\frac{\partial}{\partial x_{2}} \psi_{1}-\frac{\partial}{\partial x_{1}} \psi_{2}\right)+(\sigma+1) \sin \theta\left(\frac{\partial}{\partial x_{1}} \psi_{1}+\frac{\partial}{\partial x_{2}} \psi_{2}\right) \\
& =(\sigma-1) \cos \theta\left(\frac{\partial}{\partial x_{2}} \psi_{1}+\frac{\partial}{\partial x_{1}} \psi_{2}\right)+(\sigma-1) \sin \theta\left(\frac{\partial}{\partial x_{1}} \psi_{1}-\frac{\partial}{\partial x_{2}} \psi_{2}\right) \\
& -(\sigma+1) \sin \theta\left(\frac{\partial}{\partial x_{2}} \psi_{1}-\frac{\partial}{\partial x_{1}} \psi_{2}\right)+(\sigma+1) \cos \theta\left(\frac{\partial}{\partial x_{1}} \psi_{1}+\frac{\partial}{\partial x_{2}} \psi_{2}\right) \\
& =(\sigma-1) \sin \theta\left(\frac{\partial}{\partial x_{2}} \psi_{1}+\frac{\partial}{\partial x_{1}} \psi_{2}\right)-(\sigma-1) \cos \theta\left(\frac{\partial}{\partial x_{1}} \psi_{1}-\frac{\partial}{\partial x_{2}} \psi_{2}\right)
\end{aligned}
$$

i.e.,

$$
\begin{aligned}
\cos \theta \frac{\partial}{\partial x_{2}} \psi_{1}+\sigma \sin \theta \frac{\partial}{\partial x_{2}} \psi_{2} & =-\sin \theta \frac{\partial}{\partial x_{1}} \psi_{1}+\sigma \cos \theta \frac{\partial}{\partial x_{1}} \psi_{2} \\
-\sigma \sin \theta \frac{\partial}{\partial x_{2}} \psi_{1}+\cos \theta \frac{\partial}{\partial x_{2}} \psi_{2} & =-\sigma \cos \theta \frac{\partial}{\partial x_{1}} \psi_{1}-\sin \theta \frac{\partial}{\partial x_{1}} \psi_{2}
\end{aligned}
$$


or, equivalently, in the form (42), as desired.

Corollary 3. Deformations of the metric (30) give rise to the deformations of the supercomplex structure described by the Fueter equation (39) with $\gamma$ given by $(38)$.

COROLlary 4. Theorems 5 and 6 in [12] hold true for quasiconformal mappings with arbitrary complex dilatations.

Remark 6. The assertion of Corollary 4 has already been known (cf. e.g. [19]), but now it is proved with the use of a new, simpler method related to the theory of isospectral deformations, just as in [12] where the same was done in the particular case of real-valued complex dilatations.

\section{References}

[1] M. J. Ablowitz, D. J. Kaup, A. C. Newell and H. Seguer, The inverse scattering transform-Fourier analysis for nonlinear problems, Stud. Appl. Math. 53 (1974), 245-315.

[2] J. Adem and J. Ławrynowicz, Construction of normed maps $\mathbb{R}^{11} \times \mathbb{R}^{11} \rightarrow \mathbb{R}^{26}$, preprint, Dep. de Mat., Centro de Investigación y de Estudios Avanzados, México 1990.

[3] J. Adem, J. Ławrynowicz and J. Rembieliński, Generalized Hurwitz maps of the type $S \times V \rightarrow W$, preprint, Dep. de Mat., Centro de Investigación y de Estudios Avanzados, México 1990.

[4] M. F. Atiyah, Geometry of Yang-Mills Fields, Lezioni Fermione, Academia Nazionale dei Lincei-Scuola Normale Superiore, Pisa 1979.

[5] E. Date, M. Jimbo, M. Kashiwara and T. Miwa, Transformation groups for soliton equations, in: Proc. RIMS Symp. Nonlinear Integrable Systems-Classical and Quantum Theory, Kyoto 1981, World Scientific, Singapore 1983, 39-119.

[6] R. Fueter, Über die analytische Darstellung der regulären Funktionen einer Quaternionenvariablen, Comment. Math. Helv. 8 (1936), 371-381.

[7] I. Fukuroya, S. Kanemaki and O. Suzuki, Hermitian Hurwitz pairs, preprint, Nihon Univ., Dept. of Math., 1988, 26 pp.

[8] M. B. Green, J.H. Schwarz and E. Witten, Superstring Theory I-II, Cambridge Monographs Math. Phys., Cambridge Univ. Press, Cambridge 1987.

[9] A. Hurwitz, Über die Komposition der quadratischen Formen von beliebig vielen Variablen, Nachr. Königl. Gesell. Wiss. Göttingen Math.-Phys. Kl. 1898, 309-316; reprinted in : A. Hurwitz, Mathematische Werke II, Birkhäuser, Basel 1933, 565571.

[10] —, Über die Komposition der quadratischen Formen, Math. Ann. 88 (1923), 125; reprinted in: A. Hurwitz, Mathematische Werke II, Birkhäuser, Basel 1933, 641-666.

[11] J. Kalina, J. Ławrynowicz and O. Suzuki, A field equation defined by a Hurwitz pair, Proc. 13th Winter School on Abstract Analysis, Srní (Bohemian Forest), Rend. Circ. Mat. Palermo (2) Suppl. 9 (1985), 117-128.

[12] - , - - , Partial differential equations connected with some Clifford structures and the related quasiconformal mappings, Rend. Sem. Fac. Sci. Univ. Cagliari 57 (1987), $131-142$. 
[13] S. Kanemaki, Hurwitz pairs and octonions, in: Deformations of Mathematical Structures. Complex Analysis and Physical Applications, Selected papers from the Seminar on Deformations, Łódź-Lublin 1985/87, J. Ławrynowicz (ed.), Kluwer, Dordrecht 1989, 215-223.

[14] S. Kanemaki and O. Suzuki, Hermitian pre-Hurwitz pairs and the Minkowski space, ibid., 225-232.

[15] K. Kędzia, Hyperfine interactions versus intrinsic symmetries of many-electron systems in near-surface regions, Acta Phys. Superficiaerum 2 (1991), to appear.

[16] W. Królikowski, On correspondence between equations of motion for Dirac particle in curved and twisted space-times, ibid. 57 (1987), 143-153.

[17] -, Anisotropic complex structure on pseudo-Euclidean Hurwitz pairs, this volume, $225-240$.

[18] G. L. Lamb, Jr., Elements of Soliton Theory, Wiley, New York 1980.

[19] J. Ławrynowicz (in cooperation with J. Krzyż), Quasiconformal Mappings in the Plane. Parametrical Methods, Lecture Notes in Math. 978, Springer, Berlin 1983.

[20] J. Lawrynowicz and J. Rembieliński, Hurwitz pairs equipped with complex structures, in: Seminar on Deformations, Proc. Łódź-Warsaw 1982/84. J. Ławrynowicz (ed.), Lecture Notes in Math. 1165, Springer, Berlin 1985, 184-195.

[21] —, - Supercomplex vector spaces and spontaneous symmetry breaking, in: Seminari di Geometria 1984, Universitá di Bologna, Bologna 1985, 131-154.

[22] —, - Pseudo-euclidean Hurwitz pairs and generalized Fueter equations, in: Clifford Algebras and Their Applications in Mathematical Physics, Proc. Canterbury 1985, J.S.R. Chisholm and A.K. Common (eds.), Reidel, Dordrecht 1986, 39-48.

[23] - - - Complete classification for pseudo-euclidean Hurwitz pairs including the symmetry operations, Bull. Soc. Sci. Lettres Lódź 36 no. 29 (Série: Recherches sur les déformations, Volume "50 Years Anniversary of Scientific Work of Zygmunt Charzyński", 4 no. 39) (1986), 13 pp.

[24] - , - Pseudo-euclidean Hurwitz pairs and the Katuża-Klein theories, J. Phys. A. Math. Gen. 20 (1987), 5831-5848.

[25] - - - On the composition of nondegenerate quadratic forms with an arbitrary index, Ann. Fac. Sci. Toulouse Math. (5) 10 (1989), 141-168 (due to a printing error in vol. 10, the whole article was reprinted in vol. 11 (1990), no. 1 of the same journal, pp. 141-168).

[26] M. Mulase, Complete integrability of the Kadomtsev-Petviashvili equation, Adv. in Math. 54 (1984), 57-66.

[27] M. Ohtsuka, Dirichlet Problem, Extremal Length and Prime Ends, Van NostrandReinhold, New York 1970.

[28] —, Extremal length and precise functions in 3-space, Lecture Notes, Department of Math., Hiroshima Univ., Hiroshima 1973.

[29] R. Sasaki and I. Yamanaka, Virasoro algebra, vertex operators, quantum sineGordon and solvable quantum field theories, in: Conformal Field Theory and Solvable Lattice Models, Proc. Nagoya 1987, M. Jimbo, T. Miwa and A. Tsuchiya (eds.), Adv. Stud. in Pure Math. 16, Nagoya Univ. Press, Nagoya 1987.

[30] M. Sato, Soliton equations and Grassmann manifolds, lectures delivered at Nagoya Univ., 1982.

[31] J. H. Schwarz, Superstring theory, Phys. Rep. 89 (1982), 223-322. 
[32] O. Suzuki, J. Ławrynowicz, J. Kalina and S. Kanemaki, A geometric approach to the Kadomtsev-Petviashvili system I-II, Proc. Inst. Nat. Sci. College Hum. Sci. Nihon Univ. 21 (1986), 11-34 and 24 (1989), to appear.

INSTITUTE OF MATHEMATICS

POLISH ACADEMY OF SCIENCES

ŁÓDŹ BRANCH

NARUTOWICZA 56

90-136 ŁÓDŹ, POLAND
DEPARTMENT OF MATHEMATICS COLLEGE OF HUMANITIES AND SCIENCES NIHON UNIVERSITY TOKYO 156, JAPAN

Reçu par la Rédaction le 14.9.1990 